\title{
A nonlinear extension of the spin-2 partially massless symmetry
}

\author{
Sebastian Garcia-Saenz and Rachel A. Rosen \\ Physics Department and Institute for Strings, Cosmology, and Astroparticle Physics, \\ Columbia University, New York, NY 1002\%, U.S.A. \\ E-mail: sgsaenz@phys.columbia.edu, rar2172@columbia.edu
}

ABSTRACT: We investigate the possibility of extending the "partially massless" symmetry of a spin-2 field in de Sitter to nonlinear order. To do so, we impose a closure condition on the symmetry transformations. This requirement imposes strong constraints on the form of the nonlinear symmetry while making only minimal assumptions about the form of the nonlinear partially massless action. We find a unique nonlinear extension of the free partially massless symmetry. However, we show that no consistent Lagrangian that contains at most two derivatives of the fields can realize this symmetry.

Keywords: Gauge Symmetry, Classical Theories of Gravity

ARXIV EPRINT: 1410.8734 


\section{Contents}

1 Introduction and summary 1

$\begin{array}{llr}2 & \text { Background } & 3\end{array}$

3 Closure condition on the PM gauge symmetry 4

3.1 General argument 4

3.2 Imposing the closure condition 5

$\begin{array}{lll}3.3 & \text { Final results } & 7\end{array}$

$\begin{array}{lll}4 & \text { The action } & 7\end{array}$

$\begin{array}{llr}5 & \text { Discussion } & 9\end{array}$

\section{Introduction and summary}

A massive graviton on de Sitter spacetime can acquire an enhanced scalar gauge symmetry for a special choice of the graviton mass relative to the de Sitter curvature. The symmetry removes the helicity-0 mode of the graviton, leaving one fewer physical degree of freedom. This theory is known as partially massless (PM) gravity [1-4]. It enjoys a number of compelling properties such as stability [5, 6], null propagation [7], extensions to Einstein backgrounds [8], as well as conformal invariance in four dimensions $[9,10]$ and a remarkable analogy to electromagnetism [11-13]. In addition, because the PM gauge symmetry ties the value of the cosmological constant to the mass of the graviton, which is in turn technically natural, the PM theory offers a tantalizing new approach to the cosmological constant problem.

The field theory that describes this particle is known at the linear level. However, the linear theory is not phenomenologically viable and thus we are motivated to search for a nonlinear completion of this theory. This question has recently received much attention [1425]. In part, this is due to the recent success in constructing nonlinear ghost-free theories of massive gravity [26-30] (see, [31, 32] for reviews). Yet despite this progress and some encouraging findings, solid no-go results exist in the literature. In $D=4$ dimensions (and only $D=4$ ) there exists a two-derivative cubic Lagrangian with a nonlinear partially massless symmetry [14, 23]. However, in arbitrary spacetime dimensions, no two-derivative quartic Lagrangian exists [14]. In particular, among the new nonlinear ghost-free massive gravity theories, no PM theory exists [20, 23].

Thus certain candidate nonlinear PM theories have been ruled out. While these theories were constructed using reasonable assumptions, they are, in fact, not the most general theories one can consider. The aim of this work is to derive a conclusive "go" or "no-go" 
result for the nonlinear PM theory while making no assumptions about the form of the Lagrangian. To do so, we will take a somewhat different route from that of previous works. We will focus on the candidate symmetries rather on the candidate Lagrangians of PM gravity. The main tool we will use is a consistency condition on the nonlinear symmetry: we will demand that the symmetry forms an algebra. In other words, the commutator of two successive transformations should itself be a transformation. In this way, we can determine the possible nonlinear extensions of the PM gauge symmetry, without making any a priori assumptions about the form of the action. Our analysis largely follows that of Wald [33-35] who used this approach to derive the nonlinear symmetries of massless spin-1 and spin-2 fields.

In particular, our starting point is the partially massless symmetry of the free theory:

$$
\delta h_{\mu \nu}^{(0)}=\left(\bar{\nabla}_{\mu} \bar{\nabla}_{\nu}+H^{2} \bar{g}_{\mu \nu}\right) \phi(x) .
$$

We consider a generic nonlinear extension of this symmetry of the form

$$
\delta h_{\alpha \beta}=B_{\alpha \beta}^{\mu \nu}\left(\bar{\nabla}_{\mu} \bar{\nabla}_{\nu}+D_{\mu \nu}^{\lambda} \bar{\nabla}_{\lambda}+C_{\mu \nu}\right) \phi(x) .
$$

The $B, C$, and $D$ tensors are functions of the field $h_{\mu \nu}$ and its derivatives and must reduce to (1.1) at lowest order in the fields. The main assumption of this paper is the number of derivatives that appear in the gauge transformation, which we limit to be two. Thus $B_{\alpha \beta}^{\mu \nu}$ contains no derivatives, $D_{\mu \nu}^{\lambda}$ contains one, and $C_{\mu \nu}$ contains at most two.

Consistency requires that the nonlinear partially massless symmetry form an algebra:

$$
\left[\delta_{\phi}, \delta_{\psi}\right] h_{\alpha \beta}=\delta_{\chi} h_{\alpha \beta} .
$$

We impose this condition on (1.2) and solve for $B, C$, and $D$ order by order. We find a unique nonlinear extension of the PM symmetry:

$$
\delta h_{\alpha \beta}=\left(\bar{\nabla}_{\alpha} \bar{\nabla}_{\beta}+H^{2} \bar{g}_{\alpha \beta}\right) \phi+\gamma \bar{g}_{\alpha \beta}\left[F^{\lambda \mu \nu} F_{\lambda \mu \nu}-\frac{2}{(D-1)} F_{\mu}^{\lambda \mu} F_{\lambda \nu}{ }^{\nu}\right] \phi
$$

where $F_{\lambda \mu \nu} \equiv \bar{\nabla}_{\lambda} h_{\mu \nu}-\bar{\nabla}_{\mu} h_{\lambda \nu}$ and $\gamma$ is a free parameter.

The existence of such a symmetry does not guarantee an invariant Lagrangian. In section 4 we perform a brute force analysis and show that no consistent Lagrangian that contains at most two derivatives can realize this symmetry. This is both consistent with previous findings and generalizes them, as we are able to exclude the possibility of two derivative Lagrangians that contain, say, no cubic or quartic terms but are still able to realize a nonlinear PM symmetry at higher order. We discuss further implications of our results in section 5 .

For clarity, we present only our main results here and omit many of the derivations. Readers interested in further details can refer to the arXiv version of this paper.

Conventions. Our choice for the metric signature is $\eta_{\mu \nu}=\operatorname{diag}(-,+,+,+, \ldots)$. We assume throughout that the number of spacetime dimensions is $D \geq 3$. Symmetrizations and antisymmetrizations of indices are defined with unit weight. 


\section{Background}

The dynamics of a free massive spin- 2 field $h_{\mu \nu}$ on a $D$-dimensional de Sitter background is described by the Fierz-Pauli action:

$$
\begin{aligned}
S=\int d^{D} x \sqrt{-\bar{g}}[ & -\frac{1}{2} \bar{\nabla}_{\lambda} h^{\mu \nu} \bar{\nabla}^{\lambda} h_{\mu \nu}+\bar{\nabla}_{\lambda} h^{\mu \nu} \bar{\nabla}_{\mu} h_{\nu}^{\lambda}-\bar{\nabla}_{\mu} h \bar{\nabla}_{\nu} h^{\mu \nu}+\frac{1}{2} \bar{\nabla}^{\mu} h \bar{\nabla}_{\mu} h \\
& \left.+\frac{\bar{R}}{D}\left(h^{\mu \nu} h_{\mu \nu}-\frac{1}{2} h^{2}\right)-\frac{m^{2}}{2}\left(h^{\mu \nu} h_{\mu \nu}-h^{2}\right)\right] .
\end{aligned}
$$

Here $\bar{g}_{\mu \nu}, \bar{\nabla}_{\mu}$ and $\bar{R}$ are the metric, covariant derivative and curvature of the de Sitter background. The helicity-1 components of the field are stable whenever the graviton mass, $m$, satisfies the inequality $m^{2}>0[11]$. The helicity- 0 component is stable provided that $m$ satisfies the Higuchi bound [5]:

$$
m^{2}>\frac{(D-2)}{D(D-1)} \bar{R}
$$

When the Higuchi bound is saturated, the action boasts a scalar gauge symmetry of the form

$$
\delta h_{\mu \nu}=\left(\bar{\nabla}_{\mu} \bar{\nabla}_{\nu}+\frac{m^{2}}{(D-2)} \bar{g}_{\mu \nu}\right) \phi(x) .
$$

This symmetry removes the helicity-0 mode, rendering the number of degrees of freedom to be $D(D-1) / 2-2$. This is the free partially massless $(\mathrm{PM})$ theory, whose action is given explicitly by

$$
\begin{aligned}
S=\int d^{D} x \sqrt{-\bar{g}}[ & -\frac{1}{2} \bar{\nabla}_{\lambda} h^{\mu \nu} \bar{\nabla}^{\lambda} h_{\mu \nu}+\bar{\nabla}_{\lambda} h^{\mu \nu} \bar{\nabla}_{\mu} h_{\nu}^{\lambda}-\bar{\nabla}_{\mu} h \bar{\nabla}_{\nu} h^{\mu \nu}+\frac{1}{2} \bar{\nabla}^{\mu} h \bar{\nabla}_{\mu} h \\
& \left.+\frac{H^{2}}{2}\left(D h^{\mu \nu} h_{\mu \nu}-h^{2}\right)\right] .
\end{aligned}
$$

We have chosen to write $m^{2}$ and $\bar{R}$ in terms of the Hubble constant $H$ via the relations (including the cosmological constant $\Lambda$ for later use)

$$
H^{2}=\frac{\bar{R}}{D(D-1)}=\frac{m^{2}}{(D-2)}=\frac{2 \Lambda}{(D-1)(D-2)} .
$$

While the PM symmetry exists in AdS as well, having $\bar{R}>0$ ensures the stability of the remaining modes.

The partially massless Lagrangian can be written in terms of an invariant field strength tensor $[11,36]$ :

$$
F_{\lambda \mu \nu} \equiv \bar{\nabla}_{\lambda} h_{\mu \nu}-\bar{\nabla}_{\mu} h_{\lambda \nu} .
$$

Using the notation $F_{\lambda} \equiv \bar{g}^{\mu \nu} F_{\lambda \mu \nu}$, the action (2.4) becomes

$$
S=-\frac{1}{4} \int d^{D} x \sqrt{-\bar{g}}\left[F^{\lambda \mu \nu} F_{\lambda \mu \nu}-2 F^{\lambda} F_{\lambda}\right] .
$$

In fact, any action constructed out of $F_{\lambda \mu \nu}$ and (de Sitter) covariant derivatives of $F_{\lambda \mu \nu}$ will be exactly invariant under the symmetry transformation (2.3). In particular, we can 
consider nonlinear theories which contain higher powers of $F$. However, the particular choice of terms in (2.7) guarantees the theory propagates the right number of degrees of freedom for the partially massless theory and contains no ghosts.

As a free theory, PM gravity (2.7) is consistent. However, the recent success in constructing nonlinear theories of massive gravity has prompted the search for a nonlinear PM theory of which the action in (2.4) is but the lowest order term (in a power series in $h_{\mu \nu}$ ), and of which the transformation in (2.3) is only the $h$-independent part of a nonlinear infinitesimal gauge symmetry. Finding an extension of this symmetry is the focus of this paper.

\section{Closure condition on the PM gauge symmetry}

\subsection{General argument}

Our goal is to extend the lowest order partially massless gauge symmetry (2.3) to a fully nonlinear (in $h_{\mu \nu}$ ), two-derivative scalar gauge symmetry. Generically, this symmetry can be written as

$$
\delta_{\phi} h_{\alpha \beta}=B_{\alpha \beta}^{\mu \nu}\left(\bar{\nabla}_{\mu} \bar{\nabla}_{\nu} \phi+D_{\mu \nu}^{\lambda} \bar{\nabla}_{\lambda} \phi+C_{\mu \nu} \phi\right) .
$$

Here $B_{\alpha \beta}^{\mu \nu}=B_{\alpha \beta}^{(\mu \nu)}=B_{(\alpha \beta)}^{\mu \nu}$ contains no derivatives of $h_{\mu \nu} ; D_{\mu \nu}^{\lambda}=D_{(\mu \nu)}^{\lambda}$ contains a single derivative of $h_{\mu \nu}$; and $C_{\mu \nu}=C_{(\mu \nu)}$ contains terms linear in $\bar{\nabla} \bar{\nabla} h$, quadratic in $\bar{\nabla} h$, and terms with no derivatives. The assumption that the gauge transformation contains at most two derivatives is perhaps the most important restriction in our argument. At the zeroth order we have, by assumption,

$$
B_{\alpha \beta}^{(0) \mu \nu}=\delta_{(\alpha}^{\mu} \delta_{\beta)}^{\nu}, \quad D_{\alpha \beta}^{(0) \lambda}=0, \quad C_{\alpha \beta}^{(0)}=H^{2} \bar{g}_{\alpha \beta} .
$$

We adopt as a criterion of consistency that the act of two subsequent symmetry transformations must itself constitute a symmetry transformation. In other words, for this infinitesimal symmetry to be "integrable", it must satisfy the closure condition that for any two gauge parameters $\phi$ and $\psi$ the following equation must hold for some function $\chi$,

$$
\left[\delta_{\phi}, \delta_{\psi}\right] h_{\alpha \beta}=\delta_{\chi} h_{\alpha \beta} .
$$

This condition places very strong constraints on the form of the nonlinear symmetry (3.1). Our goal is to solve this equation for the unknown tensors $B_{\alpha \beta}^{\mu \nu}, D_{\mu \nu}^{\lambda}$ and $C_{\mu \nu}$ as series in $h_{\mu \nu}$. To do so write these tensors as well as the unknown gauge function $\chi$ as power series in $h_{\mu \nu}$ and examine eq. (3.3) order by order in $h_{\mu \nu}$, to solve for $B_{\alpha \beta}^{\mu \nu}, D_{\mu \nu}^{\lambda}$ and $C_{\mu \nu}$.

Before proceeding, we note that there are several ways in which a nonlinear symmetry may be a trivial rewriting of the lowest order symmetry: if it arises from a redefinition of the gauge parameter or if it arises from a redefinition of $h_{\mu \nu}$. In the first case, a redefinition of the gauge parameter $\phi \rightarrow f \phi$ can be absorbed by a redefinition of the tensors

$$
\begin{aligned}
B^{\mu \nu}{ }_{\alpha \beta} & \rightarrow f B^{\mu \nu}{ }_{\alpha \beta}, \\
D^{\lambda}{ }_{\alpha \beta} & \rightarrow D_{\alpha \beta}^{\lambda}+2 f^{-1} \delta_{(\alpha}^{\lambda} \bar{\nabla}_{\beta)} f, \\
C_{\alpha \beta} & \rightarrow C_{\alpha \beta}+f^{-1} \bar{\nabla}_{\alpha} \bar{\nabla}_{\beta} f+f^{-1} D^{\lambda}{ }_{\alpha \beta} \bar{\nabla}_{\lambda} f .
\end{aligned}
$$


where $f$ is an arbitrary function constructed from $h_{\mu \nu}$, with $\left.f\right|_{h=0}=1$. In what follows we will use the redefinitions in (3.4) to eliminate spurious nonlinear symmetries.

In addition, redefinitions of the field $h_{\mu \nu}$ can also lead to trivial nonlinear symmetries. Suppose we perform an algebraic field redefinition $h_{\mu \nu} \rightarrow \widetilde{h}_{\mu \nu}\left(h_{\lambda \sigma}\right)$. The EOM then changes as

$$
\mathcal{E}^{\alpha \beta} \rightarrow \widetilde{\mathcal{E}}^{\alpha \beta} \equiv \frac{\delta S}{\delta \widetilde{h}_{\alpha \beta}}=\mathcal{E}^{\lambda \sigma} \frac{\partial h_{\lambda \sigma}}{\partial \widetilde{h}_{\alpha \beta}} .
$$

We see that certain terms in $B_{\alpha \beta}^{\mu \nu}$ can be absorbed by field redefinitions of $h_{\mu \nu}$. We use this freedom of redefinition to simplify our expressions in the following sections.

Finally, we note that the lowest order partially massless symmetry (2.3) closes trivially by itself. From a symmetry point of view, it is consistent to have nonlinear PM actions constructed out of the invariants $F_{\mu \nu \lambda}$ and derivatives of $F_{\mu \nu \lambda}$ that are exactly invariant under (2.3). (Though these actions are not guaranteed to be ghost-free.) Here, we look instead for nonlinear extensions of (2.3).

\subsection{Imposing the closure condition}

We start by using the closure condition to constrain the possible first-order corrections to the partially massless symmetry. We consider the most general order-one $B, D$ and $C$ tensors. The coefficients of these terms must obey the closure condition at zeroth order in $h_{\mu \nu}$. We have four independent quadratic field redefinitions and another trivial tensor $B^{(1)}$ comes from a redefinition of the gauge parameter. We find six independent solutions for the tensors $D^{(1)}$ and $C^{(1)}$, all of which can be written in terms of the invariant tensor $F$ given in (2.6):

$$
\begin{aligned}
B_{\alpha \beta}^{(1) \mu \nu} & =0 \\
D_{\alpha \beta}^{(1) \lambda} & =\alpha_{1} F_{(\alpha \beta)}^{\lambda}+\alpha_{2} \bar{g}_{\alpha \beta} F^{\lambda}+\alpha_{3} \delta_{(\alpha}^{\lambda} F_{\beta)}, \\
C_{\alpha \beta}^{(1)} & =\beta_{1} \bar{\nabla}_{\rho} F_{(\alpha \beta)}^{\rho}+\beta_{2} \bar{g}_{\alpha \beta} \bar{\nabla}_{\rho} F^{\rho}+\beta_{3} \bar{\nabla}_{(\alpha} F_{\beta)} .
\end{aligned}
$$

These are simply all the possible expressions such that $\delta_{\phi}^{(0)} D_{\alpha \beta}^{(1) \lambda}=0$ and $\delta_{\phi}^{(0)} C_{\alpha \beta}^{(1)}=0$. And so

$$
\delta_{\chi^{(0)}}^{(0)} h_{\alpha \beta}=\left(\bar{\nabla}_{\alpha} \bar{\nabla}_{\beta}+H^{2} \bar{g}_{\alpha \beta}\right) \chi^{(0)}=0 .
$$

Thus $\chi^{(0)}$ is independent of the gauge parameters $\phi$ and $\psi$.

Some of the terms in (3.6) lead to trivial symmetries in the sense that they vanish on the linear EOM. Taking this into consideration, the most general nontrivial tensors $B^{(1)}$, $D^{(1)}$ and $C^{(1)}$ satisfying the zeroth order closure condition are given by

$$
\begin{aligned}
B_{\alpha \beta}^{(1) \mu \nu} & =0, \\
D_{\alpha \beta}^{(1) \lambda} & =\alpha_{1} F_{(\alpha \beta)}^{\lambda}+\alpha_{2} \bar{g}_{\alpha \beta} F^{\lambda}+\alpha_{3} \delta_{(\alpha}^{\lambda} F_{\beta)}, \\
C_{\alpha \beta}^{(1)} & =\beta_{1} \bar{\nabla}_{\rho} F_{(\alpha \beta)}^{\rho} .
\end{aligned}
$$

We can make contact with results known in the literature. We see that the $D=4$ nonlinear partially massless symmetry found in $[14,23,24]$ is a specific case of the coefficients 
given above, in particular when $\alpha_{1} \neq 0$ and all other coefficients are zero. It is interesting to note that this symmetry also appears in a truncation of Weyl gravity in $D=4$ [10], which is fourth order in derivatives. The degrees of freedom of Weyl gravity on a de Sitter background correspond to those of a massless graviton plus a PM spin-2 [37]; setting the metric to be a nondynamical Einstein manifold yields a theory that is PM invariant up to cubic order in interactions. However, we will see that this symmetry does not survive the imposition of the closure condition at higher-orders.

We now turn to the quadratic terms in the gauge symmetry $B^{(2)}, D^{(2)}$ and $C^{(2)}$. We find that the closure condition forces the linear terms $D^{(1)}$ and $C^{(1)}$ to vanish:

$$
\begin{aligned}
D_{\alpha \beta}^{(1) \lambda} & =0, \\
C_{\alpha \beta}^{(1)} & =0 .
\end{aligned}
$$

In particular, the nonlinear symmetry found for $D=4$ in [23] does not survive the higher order closure argument. In addition, we find that $B^{(2)}$ and $D^{(2)}$ must vanish, while tensor $C^{(2)}$ contains the six independent contractions with two powers of the invariant tensor $F$ :

$$
\begin{aligned}
B^{(2) \mu \nu}= & 0, \\
D^{(2) \lambda}= & 0, \\
C_{\alpha \beta}^{(2)}= & \gamma_{1} \bar{g}_{\alpha \beta} F^{\lambda} F_{\lambda}+\gamma_{2} F_{\alpha} F_{\beta}+\gamma_{3} F^{\lambda} F_{\lambda(\alpha \beta)} \\
& +\gamma_{4} \bar{g}_{\alpha \beta} F^{\lambda \mu \nu} F_{\lambda \mu \nu}+\gamma_{5} F_{\mu \nu \alpha} F_{\beta}^{\mu \nu}+\gamma_{6} F_{\alpha \mu \nu} F_{\beta}{ }^{\mu \nu} .
\end{aligned}
$$

The function $\chi^{(1)}$ must satisfy

$$
\delta_{\chi^{(1)}}^{(0)} h_{\alpha \beta}=\left(\bar{\nabla}_{\alpha} \bar{\nabla}_{\beta}+H^{2} \bar{g}_{\alpha \beta}\right) \chi^{(1)}=0 .
$$

The fact that $\delta_{\phi}^{(1)} h_{\mu \nu}=0$ now greatly simplifies the rest of the analysis. From imposing the closure condition at order three, we find the following constraint on the $C_{\alpha \beta}^{(2)}$ :

$$
\frac{\partial C_{\alpha \beta}^{(2)}}{\partial \bar{\nabla}_{\lambda} h_{\mu \nu}} C_{\mu \nu}^{(2)}=0 .
$$

This equation constrains the $\gamma$ coefficients in (3.10) so that the tensor $C_{\alpha \beta}^{(2)}$ must be given by

$$
C_{\alpha \beta}^{(2)}=\gamma_{4} \bar{g}_{\alpha \beta}\left[F^{\lambda \mu \nu} F_{\lambda \mu \nu}-\frac{2}{(D-1)} F^{\lambda} F_{\lambda}\right]
$$

At higher order, we no longer need to use a brute force approach. Following [33] one can derive a similar recursive relation and conclude that

$$
B_{\alpha \beta}^{(n+1) \mu \nu}=0, \quad D_{\mu \nu}^{(n+1) \lambda}=0, \quad C_{\mu \nu}^{(n+1)}=0,
$$

for $n \geq 2$. 


\subsection{Final results}

The full $B, D$ and $C$ tensors are

$$
\begin{aligned}
B_{\alpha \beta}^{\mu \nu} & =\delta_{(\alpha}^{\mu} \delta_{\beta)}^{\nu}, \\
D^{\lambda}{ }_{\mu \nu} & =0 \\
C_{\alpha \beta} & =H^{2} \bar{g}_{\alpha \beta}+\gamma \bar{g}_{\alpha \beta}\left[F^{\lambda \mu \nu} F_{\lambda \mu \nu}-\frac{2}{(D-1)} F^{\lambda} F_{\lambda}\right],
\end{aligned}
$$

with free parameter $\gamma$. The unique candidate infinitesimal nonlinear PM gauge symmetry is then

$$
\delta_{\phi} h_{\alpha \beta}=\left(\bar{\nabla}_{\alpha} \bar{\nabla}_{\beta}+H^{2} \bar{g}_{\alpha \beta}\right) \phi+\gamma \bar{g}_{\alpha \beta}\left[F^{\lambda \mu \nu} F_{\lambda \mu \nu}-\frac{2}{(D-1)} F^{\lambda} F_{\lambda}\right] \phi .
$$

An action that is separately invariant under the linear PM symmetry and a conformallike transformation of the form $\delta_{\psi}^{c} h_{\alpha \beta}=\bar{g}_{\alpha \beta} \psi$ would trivially be invariant under this symmetry. We note that the combination

$$
F^{\lambda \mu \nu} F_{\lambda \mu \nu}-\frac{2}{(D-1)} F^{\lambda} F_{\lambda}
$$

in addition to being a PM invariant, is also invariant under the conformal-like transformations and is thus itself invariant under the transformation (3.16). However, such a term is itself not a viable Lagrangian, since it doesn't have the ghost-free form of the PM theory (2.7). We emphasize that the nonlinear symmetry is not a trivial extension of the lowest order PM symmetry, in the sense that it is not obeyed by the free PM theory (2.7).

\section{The action}

What sort of consistent action can realize such a symmetry? The existence of a scalar gauge symmetry of the form

$$
\delta h_{\mu \nu}=\hat{P}_{\mu \nu} \phi,
$$

where $\hat{P}_{\mu \nu}$ is an operator constructed locally from $h_{\mu \nu}$, implies that the equation of motion $(\mathrm{EOM}) \mathcal{E}^{\mu \nu} \equiv \delta S / \delta h_{\mu \nu}$ must satisfy a corresponding Bianchi identity:

$$
\hat{O}_{\mu \nu} \mathcal{E}^{\mu \nu}=0
$$

where the operator $\hat{O}_{\mu \nu}$ is obtained from $\hat{P}_{\mu \nu}$ (and vice versa) by integration by parts. Let us examine the full Bianchi identity that follows from (3.16):

$$
\bar{\nabla}_{\mu} \bar{\nabla}_{\nu}\left(B^{\mu \nu}{ }_{\alpha \beta} \mathcal{E}^{\alpha \beta}\right)-\bar{\nabla}_{\lambda}\left(B^{\mu \nu}{ }_{\alpha \beta} D_{\mu \nu}^{\lambda} \mathcal{E}^{\alpha \beta}\right)+B^{\mu \nu}{ }_{\alpha \beta} C_{\mu \nu} \mathcal{E}^{\alpha \beta}=0
$$

Considering this expression perturbatively, we observe,

$$
\begin{aligned}
\left(\bar{\nabla}_{\alpha} \bar{\nabla}_{\beta}+H^{2} \bar{g}_{\alpha \beta}\right) \mathcal{E}^{(2) \alpha \beta} & =0, \\
\left(\bar{\nabla}_{\alpha} \bar{\nabla}_{\beta}+H^{2} \bar{g}_{\alpha \beta}\right) \mathcal{E}^{(k+2) \alpha \beta} & =-\gamma \widetilde{C}^{(2)} \bar{g}_{\alpha \beta} \mathcal{E}^{(k) \alpha \beta},
\end{aligned}
$$


for $k \geq 1$ and $\widetilde{C}^{(2)}=F^{\lambda \mu \nu} F_{\lambda \mu \nu}-\frac{2}{(D-1)} F^{\lambda} F_{\lambda}$. Note that $\gamma$ plays the role of a dimensionless coupling constant, as the terms with higher powers of $h_{\mu \nu}$ are proportional to higher powers of $\gamma$.

Consider the Bianchi identity that constrains the cubic EOM. If we take the lowest order Lagrangian to be the free, ghost-free PM theory (2.6), the Bianchi identity reads explicitly

$$
\left(\bar{\nabla}_{\alpha} \bar{\nabla}_{\beta}+H^{2} \bar{g}_{\alpha \beta}\right) \mathcal{E}^{(3) \alpha \beta}=\gamma(D-2) \bar{\nabla}_{\sigma} F^{\sigma}\left[F^{\lambda \mu \nu} F_{\lambda \mu \nu}-\frac{2}{(D-1)} F^{\lambda} F_{\lambda}\right] .
$$

Conceivably, a two derivative action that is quartic in the fields $\mathcal{L}^{(4)}$ might be able to satisfy such an equation.

In order to check, we perform a brute force, perturbative analysis. In fact, our analysis is more general than that required by (4.5). We take as a starting point the quadratic PM action (2.6). We then consider every possible cubic and quartic interaction with at most two derivatives. In addition, we consider the most generic linear and quadratic extensions of the gauge transformation that themselves have at most two derivatives. We then determine whether a choice of coefficients exists so that eq. (4.2) can be satisfied order by order. We find that no such action exists. Since the cubic case was already considered in [14] and the quartic result was already stated there as well, we only briefly summarize our findings here:

The cubic Lagrangian $\mathcal{L}^{(3)}$ is the unique two-derivative term that satisfies the second order identity,

$$
\hat{O}_{\mu \nu}^{(0)} \frac{\delta \mathcal{L}^{(3)}}{\delta h_{\mu \nu}}+\hat{O}_{\mu \nu}^{(1)} \frac{\delta \mathcal{L}^{(2)}}{\delta h_{\mu \nu}}=0,
$$

Here $\hat{O}_{\mu \nu}^{(0)}$ is given by the lowest order PM symmetry, and $\mathcal{L}^{(2)}$ is the free PM Lagrangian. Allowing for non-canonical derivative interactions, we find only one cubic action $\mathcal{L}^{(3)}$ exists that satisfies this expression and only when $D=4$, consistent with the results of $[14,23]$.

The part of the Bianchi identity containing three powers of $h_{\mu \nu}$ is given by

$$
\hat{O}_{\mu \nu}^{(0)} \frac{\delta \mathcal{L}^{(4)}}{\delta h_{\mu \nu}}+\hat{O}_{\mu \nu}^{(1)} \frac{\delta \mathcal{L}^{(3)}}{\delta h_{\mu \nu}}+\hat{O}_{\mu \nu}^{(2)} \frac{\delta \mathcal{L}^{(2)}}{\delta h_{\mu \nu}}=0 .
$$

The generic quartic Lagrangian contains 5 zero-derivative contractions with four powers of $h_{\mu \nu}$. We choose to write the two-derivative terms in contractions of the form $h h \bar{\nabla} h \bar{\nabla} h$. There are 43 such contractions; however, five of them can be show to be redundant via identities. Thus the generic form of $\mathcal{L}^{(4)}$ contains a total of 43 free parameters. For the operator $\hat{O}_{\mu \nu}^{(2)}$ we find 4 terms with no derivatives plus 68 with two derivatives, for a total of 72 parameters to be determined. The Bianchi identity (4.7) then contains contractions with zero, two and four derivatives with three powers of $h_{\mu \nu}$. We count 16 contractions of the form $h h \bar{\nabla} \bar{\nabla} \bar{\nabla} \bar{\nabla} h, 50$ contractions of the form $h \bar{\nabla} h \bar{\nabla} \bar{\nabla} \bar{\nabla} h, 45$ contractions of the form $h \bar{\nabla} \bar{\nabla} h \bar{\nabla} \bar{\nabla} h, 65$ contractions of the form $\bar{\nabla} h \bar{\nabla} h \bar{\nabla} \bar{\nabla} h, 12$ contractions of the form $h h \bar{\nabla} \bar{\nabla} h$, 16 contractions of the form $h \bar{\nabla} h \bar{\nabla} h$, and 3 contractions of the form $h h h$. The total number of constraints is therefore 207, which involve 115 parameters $(116$ in $D=4)$.

We then find that no set of nonzero coefficients exists that solves the constraints, except for the trivial ones that arise from field redefinitions of the free PM Lagrangian. In 
particular, the cubic Lagrangian $\mathcal{L}^{(3)}$ inevitably generates an obstruction at the next order in the Bianchi identity. Furthermore, even if cubic interactions are absent, there exist no quartic interactions with up to two derivatives that exhibit a gauge symmetry.

Note that, due to the recursive relation (4.5) the absence of a quartic Lagrangian means that no nonlinear two-derivative Lagrangian can realize the nonlinear PM symmetry with $\gamma \neq 0$. In other words, one could conceive of action that is two derivatives in the fields, has no cubic or quartic terms, and yet somehow realizes a nonlinear symmetry via higher order terms. Eq. (4.5) rules out this case: this equation cannot be satisfied if $\mathcal{E}^{(3) \alpha \beta}=0$.

\section{Discussion}

The closure condition (3.3) places powerful constraints on any nonlinear extension of the partially massless symmetry, while allowing one to remain entirely agnostic about the form of the invariant Lagrangian. The basic assumption of this paper was that the partially massless symmetry itself contains no more than two derivatives of the fields. With this assumption and using the closure condition we were able to identify a unique nonlinear partially massless symmetry. We could then show that no consistent Lagrangian which contains at most two derivatives can realize this symmetry.

For the closure condition, we have demanded that two gauge symmetries close to another gauge symmetry. More generally, it's potentially consistent for the gauge symmetries to close to a gauge symmetry plus an on-shell trivial symmetry. While this is not the situation for the gauge symmetries of massless spin- 1 and spin- 2 fields, this occurs, for example, in the case of supersymmetry without auxiliary fields. ${ }^{1}$ We have checked to see if such considerations modify our results. At lowest order, generalizing the closure condition to allow for trivial on-shell symmetries leads only to new symmetry terms that are themselves on-shell trivial. However, it remains possible that this generalization could lead to a wider family of candidate symmetries at higher order.

The candidate nonlinear symmetry (3.16) has some curious properties that distinguishes it from its GR and Yang-Mills counterparts. The symmetry not only has the feature of being Abelian, $\left[\delta_{\phi}, \delta_{\psi}\right] h_{\alpha \beta}=0$, but it is also nilpotent,

$$
\delta_{\phi} \delta_{\psi} h_{\alpha \beta}=0
$$

This means that the transformation solves the closure condition in a rather trivial way despite it being nonlinear. A consequence of the nilpotency property is that the infinitesimal transformation can be trivially integrated to yield the corresponding finite gauge transformation.

Despite these simple properties, we remark again that this nonlinear symmetry is not a trivial extension of the usual PM symmetry, since the free PM theory does not obey it.

If this symmetry can at all be realized by a Lagrangian, either higher derivative terms or additional fields are required. The method we have used here gives only the form of the symmetry and tells us little else about other properties of the invariant Lagrangian, such

\footnotetext{
${ }^{1}$ We are grateful to Kurt Hinterbichler for pointing this out.
} 
its health or stability. Thus even if a higher derivative action possesses the nonlinear PM symmetry, recent results [38] cast doubt on whether such an action can be ghost-free.

Finally, we note that imposing the closure condition on the combination of the PM symmetry plus diffeomorphisms, rather than on the PM symmetry alone can potentially allow for more general symmetries than those found here. Such a condition would be appropriate for a partially massless particle coupled to gravity. Such a possibility was considered in [24]. A no-go result was obtained for an action consistent with the gauging of $\mathrm{SO}(5,1)$. Our approach is more general than the one of [24]. It's possible that the application of the approach used here might lead to a nonlinear symmetry in this case, though it will not necessarily lead to a Lagrangian. We leave this for future work.

\section{Acknowledgments}

We would like to thank Kurt Hinterbichler, Alberto Nicolis, Riccardo Rattazzi, Andrew Tolley and Bob Wald for many productive conversations. SGS and RAR are supported by DOE grant DE-SC0011941.

Open Access. This article is distributed under the terms of the Creative Commons Attribution License (CC-BY 4.0), which permits any use, distribution and reproduction in any medium, provided the original author(s) and source are credited.

\section{References}

[1] S. Deser and R.I. Nepomechie, Gauge invariance versus masslessness in de Sitter space, Annals Phys. 154 (1984) 396 [InSPIRE].

[2] S. Deser and A. Waldron, Gauge invariances and phases of massive higher spins in (A)dS, Phys. Rev. Lett. 87 (2001) 031601 [hep-th/0102166] [INSPIRE].

[3] S. Deser and A. Waldron, Partial masslessness of higher spins in (A)dS, Nucl. Phys. B 607 (2001) 577 [hep-th/0103198] [INSPIRE].

[4] G. Gabadadze and A. Iglesias, Special massive spin-2 on de Sitter space, JCAP 02 (2008) 014 [arXiv:0801.2165] [INSPIRE].

[5] A. Higuchi, Forbidden mass range for spin-2 field theory in de Sitter space-time, Nucl. Phys. B 282 (1987) 397 [inSPIRE].

[6] S. Deser and A. Waldron, Stability of massive cosmological gravitons, Phys. Lett. B 508 (2001) 347 [hep-th/0103255] [INSPIRE].

[7] S. Deser and A. Waldron, Null propagation of partially massless higher spins in (A)dS and cosmological constant speculations, Phys. Lett. B 513 (2001) 137 [hep-th/0105181] [INSPIRE].

[8] S. Deser, E. Joung and A. Waldron, Gravitational- and self-coupling of partially massless spin 2, Phys. Rev. D 86 (2012) 104004 [arXiv:1301.4181] [inSPIRE].

[9] S. Deser and A. Waldron, Conformal invariance of partially massless higher spins, Phys. Lett. B 603 (2004) 30 [hep-th/0408155] [INSPIRE]. 
[10] S. Deser, E. Joung and A. Waldron, Partial masslessness and conformal gravity, J. Phys. A 46 (2013) 214019 [arXiv:1208.1307] [InSPIRE].

[11] S. Deser and A. Waldron, Partially massless spin 2 electrodynamics, Phys. Rev. D 74 (2006) 084036 [hep-th/0609113] [InSPIRE].

[12] S. Deser and A. Waldron, $P M=$ EM? Partially massless duality invariance, Phys. Rev. D 87 (2013) 087702 [arXiv:1301.2238] [InSPIRE].

[13] K. Hinterbichler, Manifest duality invariance for the partially massless graviton, Phys. Rev. D 91 (2015) 026008 [arXiv:1409.3565] [InSPIRE].

[14] Y. Zinoviev, On massive spin 2 interactions, Nucl. Phys. B 770 (2007) 83 [hep-th/0609170] [INSPIRE].

[15] C. de Rham and S. Renaux-Petel, Massive gravity on de Sitter and unique candidate for partially massless gravity, JCAP 01 (2013) 035 [arXiv:1206.3482] [INSPIRE].

[16] S.F. Hassan, A. Schmidt-May and M. von Strauss, On partially massless bimetric gravity, Phys. Lett. B 726 (2013) 834 [arXiv: 1208.1797] [INSPIRE].

[17] E. Joung, L. Lopez and M. Taronna, Generating functions of (partially-)massless higher-spin cubic interactions, JHEP 01 (2013) 168 [arXiv:1211.5912] [INSPIRE].

[18] S.F. Hassan, A. Schmidt-May and M. von Strauss, Bimetric theory and partial masslessness with Lanczos-Lovelock terms in arbitrary dimensions, Class. Quant. Grav. 30 (2013) 184010 [arXiv: 1212.4525] [INSPIRE].

[19] E. Joung, L. Lopez and M. Taronna, On the cubic interactions of massive and partially-massless higher spins in (A)dS, JHEP 07 (2012) 041 [arXiv:1203.6578] [INSPIRE].

[20] S. Deser, M. Sandora and A. Waldron, Nonlinear partially massless from massive gravity?, Phys. Rev. D 87 (2013) 101501 [arXiv:1301.5621] [inSPIRE].

[21] S.F. Hassan, A. Schmidt-May and M. von Strauss, Higher derivative gravity and conformal gravity from bimetric and partially massless bimetric theory, arXiv:1303.6940 [INSPIRE].

[22] S. Deser, M. Sandora and A. Waldron, No consistent bimetric gravity?, Phys. Rev. D 88 (2013) 081501 [arXiv: 1306.0647] [INSPIRE].

[23] C. de Rham, K. Hinterbichler, R.A. Rosen and A.J. Tolley, Evidence for and obstructions to nonlinear partially massless gravity, Phys. Rev. D 88 (2013) 024003 [arXiv:1302.0025] [INSPIRE].

[24] E. Joung, W. Li and M. Taronna, No-go theorems for unitary and interacting partially massless spin-two fields, Phys. Rev. Lett. 113 (2014) 091101 [arXiv:1406.2335] [InSPIRE].

[25] S.F. Hassan, A. Schmidt-May and M. von Strauss, Particular solutions in bimetric theory and their implications, Int. J. Mod. Phys. D 23 (2014) 1443002 [arXiv:1407.2772] [INSPIRE].

[26] C. de Rham and G. Gabadadze, Generalization of the Fierz-Pauli action, Phys. Rev. D 82 (2010) 044020 [arXiv: 1007.0443] [InSPIRE].

[27] C. de Rham, G. Gabadadze and A.J. Tolley, Resummation of massive gravity, Phys. Rev. Lett. 106 (2011) 231101 [arXiv:1011.1232] [INSPIRE].

[28] S.F. Hassan and R.A. Rosen, Resolving the ghost problem in non-linear massive gravity, Phys. Rev. Lett. 108 (2012) 041101 [arXiv:1106.3344] [INSPIRE]. 
[29] C. de Rham, G. Gabadadze and A.J. Tolley, Helicity decomposition of ghost-free massive gravity, JHEP 11 (2011) 093 [arXiv:1108.4521] [INSPIRE].

[30] C. de Rham, G. Gabadadze and A.J. Tolley, Ghost free massive gravity in the Stückelberg language, Phys. Lett. B 711 (2012) 190 [arXiv:1107.3820] [INSPIRE].

[31] K. Hinterbichler, Theoretical aspects of massive gravity, Rev. Mod. Phys. 84 (2012) 671 [arXiv: 1105.3735] [INSPIRE].

[32] C. de Rham, Massive gravity, Living Rev. Rel. 17 (2014) 7 [arXiv:1401.4173] [InSPIRE].

[33] R.M. Wald, Spin-2 fields and general covariance, Phys. Rev. D 33 (1986) 3613 [INSPIRE].

[34] C. Cutler and R.M. Wald, A new type of gauge invariance for a collection of massless spin-2 fields. I. Existence and uniqueness, Class. Quant. Grav. 4 (1987) 1267 [INSPIRE].

[35] R.M. Wald, A new type of gauge invariance for a collection of massless spin-2 fields. II. Geometrical interpretation, Class. Quant. Grav. 4 (1987) 1279 [InSPIRE].

[36] E.D. Skvortsov and M.A. Vasiliev, Geometric formulation for partially massless fields, Nucl. Phys. B 756 (2006) 117 [hep-th/0601095] [INSPIRE].

[37] J. Maldacena, Einstein gravity from conformal gravity, arXiv:1105.5632 [INSPIRE].

[38] C. de Rham, A. Matas and A.J. Tolley, New kinetic interactions for massive gravity?, Class. Quant. Grav. 31 (2014) 165004 [arXiv:1311.6485] [INSPIRE]. 